\title{
Vacuum Polarization by a Magnetic Flux in a Cosmic String Background
}

\author{
J. Spinelly ${ }^{a *}$ and E. R. Bezerra de Mello ${ }^{\mathrm{b} \dagger}$ \\ ${ }^{a}$ Departamento de Física, Universidade Estadual da Paraíba, \\ Av. Juvêncio Arruda S/N, Campina Grande, PB, Brazil \\ ${ }^{\mathrm{b}}$ Departamento de Física, Universidade Federal da Paraíba \\ Caixa Postal 5008, 58.059-970, João Pessoa, PB, Brazil
}

In this paper we analyse the vacuum polarization effects due to a magnetic flux on massless fermionic fields in a cosmic string background. Three distinct configurations of magnetic fields are considered. In all of them the magnetic fluxes are confined in a long cylindrical tube of finite radius.

\section{INTRODUCTION}

The vacuum polarization effects due to a magnetic field confined in a tube of finite radius in Minkowski spacetime were first analysed by Serebryanyi [1. A few years later, Guimarães and Linet [2] and Linet [3, calculated these effects to a charged massless scalar and fermionic fields, respectively, on a idealized cosmic string spacetime. There a magnetic field running through the line singularity was considered. As a consequence, the renormalized vacuum expectation value associated with the energy-moment tensor, $\left\langle T_{\mu \nu}(x)\right\rangle_{\text {Ren. }}$, presents contributions coming from the geometry of the spacetime and also the magnetic flux. In order to develop these calculations the respective Green functions in this physical system were obtained. More recently Sriramkumar 4] has calculated the vacuum fluctuation of current and energy densities for a massless charged scalar field around an idealized cosmic string carrying a magnetic flux. There the Green function was obtained taking into account the presence of the vector potential in the differential operator, $D_{\mu}=\partial_{\mu}-i e A_{\mu}$, which presents the advantage of calculating the two-points function without imposing any boundary condition on the filed.

The analysis of the vacuum polarization effects

\footnotetext{
*spinelly@fisica.ufpb.br

†emello@fisica.ufpb.br
}

on a massless charged scalar field by a magnetic flux confined in a cylindrical tube of finite radius in a cosmic string background have been analysed in [5]. There three distinct configurations of magnetic field below specified has been considered. In all of them the axis of the infinitely long tube of radius $R$ coincides with the cosmic string. Calculating the renormalized vacuum expectation value (VEV) of the square of the field, $\left\langle\Phi^{*}(x) \Phi(x)\right\rangle_{\text {Ren }}$, and the energy-momentum tensor, $\left\langle T_{\mu}^{\nu}(x)\right\rangle_{R e n}$, it was observed that these quantities present two contributions for each model of magnetic flux. The first are the standard ones due to the conical geometry of the spacetime and the magnetic flux. The second contributions are corrections due to the finite thickness of the radius of the tube. These extra terms provided relevant contributions for points outside the tube. Specifically, for the third model this contribution is a long-range effect, i.e., it is as relevant as the standard one up to a distance which exceed the radius of the observable universe.

Here in this paper we decided to continue this analysis, extending it to charged massless fermionic fields in the same physical system. As we shall see, the renormalized VEV of the zerozero component of the energy-momentum tensor also present two distinct contributions. Moreover due mainly to interaction of the spin degrees of freedom of the fermionic field with the magnetic flux, the corrections due to the nonzero thickness 
of the radius of the tube is composed by two terms with opposite signal. Additional couplings between the spin with the geometry are also present in this system.

The idealized model for a infinitely long straight static cosmic string spacetime can be given in cylindrical coordinates by the line element below:

$d s^{2}=-d t^{2}+d r^{2}+\alpha^{2} r^{2} d \theta^{2}+d z^{2}$,

where $\alpha=1-4 \mu$ is a parameter smaller than unity which codifies the presence of a conical twosurface $(r, \theta)$. In fact for a typical Grand Unified Theory, $\alpha=1-O\left(10^{-6}\right)$.

We shall consider the presence of a magnetic field along the $z$-direction assuming that the field has a finite range in the radial coordinate. We are particularly interested in the three models below: i) $H(r)=\frac{\phi}{\alpha \pi R^{2}} \Theta(R-r)$, homogeneous field inside;

ii) $H(r)=\frac{\phi}{2 \pi \alpha R r} \Theta(R-r)$, field proportional to $1 / r$

iii) $H(r)=\frac{\phi}{2 \pi \alpha R} \delta(r-R)$, cylindrical shell,

where $R$ is the radial extent of the tube, and $\phi$ is the total flux. The ratio of the flux by the quantum flux $\phi_{o}$, can be expressed by $\phi / \phi_{0}=N+\gamma$, where $N$ is the integer part and $0<\gamma<1$. $^{3}$

\section{SPINOR FEYNMAN PROPAGATOR}

The Feynmann propagator associated with a charged fermionic field, $S_{F}\left(x, x^{\prime}\right)$, obeys the following differential equation:

$$
(i \not \nabla+e A-M) S_{F}\left(x, x^{\prime}\right)=\frac{1}{\sqrt{-g}} \delta^{4}\left(x, x^{\prime}\right) I_{4},
$$

where $g=\operatorname{det}\left(-g_{\mu \nu}\right)$. The covariant derivative operator reads

$\not \nabla=e_{(a)}^{\mu} \gamma^{(a)}\left(\partial_{\mu}+\Gamma_{\mu}\right)$

$e_{(a)}^{\mu}$ being the vierbein satisfying the condition $e_{(a)}^{\mu} e_{(b)}^{\nu} \eta^{(a)(b)}=g^{\mu \nu}$ and $\Gamma_{\mu}$ is the spin connection given in terms of flat spacetime $\gamma$ matrices by

$\Gamma_{\mu}=-\frac{1}{4} \gamma^{(a)} \gamma^{(b)} e_{(a)}^{\nu} e_{(b) \nu ; \mu}$,

${ }^{3}$ In this paper we are considering $\hbar=G=c=1$. and

$A=e_{(a)}^{\mu} \gamma^{(a)} A_{\mu}$.

It can be shown that if a bispinor $D_{F}\left(x, x^{\prime}\right)$ satisfies the differential equation

$$
\begin{array}{r}
{\left[-\mathcal{D}^{2}+\frac{1}{4} R+i e g^{\mu \nu}\left(D_{\mu} A \nu\right)-i e \Sigma^{\mu \nu} F_{\mu \nu}+\right.} \\
\left.2 i e g^{\mu \nu} A_{\nu} \nabla_{\nu}+e^{2} g^{\mu \nu} A_{\mu} A_{\nu}+M^{2}\right] D_{F}\left(x, x^{\prime}\right) \\
=-\frac{1}{\sqrt{-g}} \delta^{4}\left(x, x^{\prime}\right) I_{4}
\end{array}
$$

with

$\Sigma^{\mu \nu}=\frac{1}{4}\left[\gamma^{\mu}, \gamma^{\nu}\right]$

being the generalized d'Alembertian operator given by

$\mathcal{D}^{2}=g^{\mu \nu} \nabla_{\mu} \nabla_{\nu}=g^{\mu \nu}\left(\partial_{\mu} \nabla_{\nu}+\Gamma_{\mu} \nabla_{\nu}-\Gamma_{\mu \nu}^{\alpha} \partial_{\alpha}\right)$,

then the spinor Feynman propagator may be written $\mathrm{as}^{4}$

$S_{F}\left(x, x^{\prime}\right)=(i \not \nabla+e A+M) D_{F}\left(x, x^{\prime}\right)$.

Now after this brief review about the calculation of spinor Feynmann propagator, let us specialize it to the cosmic string spacetime in the presence of a magnetic field along the $z$-direction. Wee shall chose the following base tetrad:

$e_{(a)}^{\mu}=\left(\begin{array}{cccc}1 & 0 & 0 & 0 \\ 0 & \cos \theta & -1 / \alpha r \sin \theta & 0 \\ 0 & \sin \theta & 1 / \alpha r \cos \theta & 0 \\ 0 & 0 & 0 & 1\end{array}\right)$.

As to the four vector potential we have

$A_{\mu}=(0,0, A(r), 0)$,

with

$A(r)=\frac{\phi}{2 \pi} a(r)$.

For the two first models, we can represent the radial function $a(r)$ by:

$a(r)=f(r) \Theta(R-r)+\Theta(r-R)$,

${ }^{4}$ In the absence of gauge field this result can be found in 6 . 
with

$f(r)=\left\{\begin{array}{cc}r^{2} / R^{2}, & \text { for the model }(i) \text { and } \\ r / R, & \text { for the model }(i i) .\end{array}\right.$

For the third model,

$a(r)=\Theta(R-r)$.

The only non-zero spin connection is

$\Gamma_{2}=\frac{i}{2}(1-\alpha) \Sigma^{3}$

and for the Christofell symbols we have:

$\Gamma_{22}^{1}=-\alpha^{2} r, \quad \Gamma_{12}^{2}=\Gamma_{21}^{1}=1 / r$.

Defining by $\mathcal{K}(x)$ the $4 \times 4$ matrix differential operator which acts on $D_{F}\left(x, x^{\prime}\right)$ in (6), for this physical system we obtain:

$$
\begin{aligned}
\mathcal{K}(x) & =-\Delta-\frac{i}{\alpha^{2} r^{2}}(1-\alpha) \Sigma^{3} \partial_{\theta}-e H(r) \Sigma^{3} \\
& +\frac{1}{4 \alpha^{2} r^{2}}(1-\alpha)^{2}-\frac{e}{\alpha^{2} r^{2}}(1-\alpha) A(r) \Sigma^{3} \\
& +\frac{2 i e}{\alpha^{2} r^{2}} A(r) \partial_{\theta}+\frac{e^{2}}{\alpha^{2} r^{2}} A^{2}(r)+M^{2},
\end{aligned}
$$

where

$\Sigma^{3}=\left(\begin{array}{cc}\sigma^{3} & 0 \\ 0 & \sigma^{3}\end{array}\right)$,

and

$\Delta=-\partial_{t}^{2}+\partial_{r}^{2}+\frac{1}{r} \partial_{r}+\frac{1}{\alpha^{2} r^{2}} \partial_{\theta}^{2}+\partial_{z}^{2}$.

We can see that the differential operator above explicitly exhibits, besides the ordinary d'Alembertian operator on the conical spacetime, four different types of interactions terms: $(i)$ the usual charge-magnetic field, (ii) the spinmagnetic field, (iii) the spin-geometry and (iv) the spin-charge-geometry. All of the last three interactions were absent in the analogous differential operator used to define the scalar Green function in [5].

Moreover as we can see the operator $\mathcal{K}(x)$ is diagonal in $2 \times 2$ blocks. This means that the two upper components of the Dirac spinor interact with the gravitational and magnetic fields in similar way as the two lower components and they do not interact among themselves.

The system that we what to study consists of massless charged fermionic field in the cosmic string spacetime in the presence of an Abelian magnetic field along the $z$-direction. Let us chose a left-handed field. In this case the Dirac equation and the equation which defines the spinor Feynman propagator reduce themselves to a $2 \times 2$ matrix differential equation as shown below:

$\not D_{L} \chi=0$,

where

$$
\begin{gathered}
\not D_{L}=i\left[\partial_{t}-\sigma^{(r)}\left(\partial_{r}-\frac{\left(\alpha^{-1}-1\right)}{2 r}\right)\right. \\
\left.-\frac{1}{\alpha r} \sigma^{(\theta)}\left(\partial_{\theta}-i A(r)\right)-\sigma^{(z)} \partial_{z}\right],
\end{gathered}
$$

with $\sigma^{(r)}=\vec{\sigma} \cdot \hat{r}, \sigma^{(\theta)}=\vec{\sigma} \cdot \hat{\theta}$ and $\sigma^{(z)}=\vec{\sigma} . \hat{z}$.

The Feynman two-components propagator obeys the equation

$i \not D_{L} S_{F}^{L}\left(x, x^{\prime}\right)=\frac{1}{\sqrt{-g}} \delta^{4}\left(x, x^{\prime}\right) I_{2}$,

and can be given by

$S_{F}^{L}\left(x, x^{\prime}\right)=i \not D_{L} G^{L}\left(x, x^{\prime}\right)$,

where now $G^{L}\left(x, x^{\prime}\right)$ obeys the $2 \times 2$ matrix differential operator below:

$\bar{K}(x) G^{L}\left(x, x^{\prime}\right)=-\frac{1}{\sqrt{-g}} \delta^{4}\left(x, x^{\prime}\right) I_{2}$,

with

$$
\begin{aligned}
\bar{K}(x) & =-\Delta-\frac{i}{\alpha^{2} r^{2}}(1-\alpha) \sigma^{3} \partial_{\theta}-e H(r) \sigma^{3} \\
& +\frac{1}{4 \alpha^{2} r^{2}}(1-\alpha)^{2}-\frac{e}{\alpha^{2} r^{2}}(1-\alpha) A(r) \sigma^{3} \\
& +\frac{2 i e}{\alpha^{2} r^{2}} A(r) \partial_{\theta}+\frac{e^{2}}{\alpha^{2} r^{2}} A^{2}(r) .
\end{aligned}
$$

Because of the peculiar diagonal form of the above operator, let us take for $G^{L}$ the following expression:

$G^{L}\left(x, x^{\prime}\right)=\left(\begin{array}{cc}G_{+}\left(x, x^{\prime}\right) & 0 \\ 0 & G_{-}\left(x, x^{\prime}\right)\end{array}\right)$. 
So the differential equation (24) reduces itself to a two independent ones as shown below:

$$
\begin{array}{r}
{\left[\Delta \pm \frac{i}{\alpha^{2} r^{2}}(1-\alpha) \partial_{\theta}-\frac{(1-\alpha)^{2}}{4 \alpha^{2} r^{2}} \pm e H(r)\right.} \\
-\frac{2 i e}{\alpha^{2} r^{2}} A(r) \partial_{\theta} \pm \frac{e}{\alpha^{2} r^{2}}(1-\alpha) A(r)- \\
\left.\frac{e^{2}}{\alpha^{2} r^{2}} A^{2}\right] G_{ \pm}\left(x, x^{\prime}\right)=\frac{1}{\sqrt{-g}} \delta^{4}\left(x, x^{\prime}\right) .
\end{array}
$$

Due to the cylindrical symmetry of this system each component of the Euclidean Green function can be expressed by

$$
\begin{array}{r}
G_{ \pm}\left(x, x^{\prime}\right)=\frac{1}{(2 \pi)^{3}} \sum_{n=-\infty}^{\infty} e^{i n\left(\theta-\theta^{\prime}\right)} \int_{-\infty}^{\infty} d k \\
\int_{-\infty}^{\infty} d \omega e^{i k\left(z-z^{\prime}\right)} e^{i \omega\left(\tau-\tau^{\prime}\right)} g_{n}^{ \pm}\left(r, r^{\prime}\right) .
\end{array}
$$

Before to specialize on the specific model let us write down the non-homogeneous differential equation obeyed by the unknown function $g_{n}^{ \pm}\left(r, r^{\prime}\right)$. Substituting (28) into (27) and using the standard representation to the delta function in the temporal, angular and z-coordinates, we arrive to following differential equation for the unknown function $g_{n}^{ \pm}\left(r, r^{\prime}\right)$ :

$$
\begin{aligned}
{\left[\frac{d^{2}}{d r^{2}}+\right.} & \frac{1}{r} \frac{d}{d r}-\frac{1}{\alpha^{2} r^{2}}\left[n^{2} \pm n(1-\alpha \mp 2 n e A)\right. \\
& \left.+\frac{\left(1-\alpha^{2}\right)}{4} \mp e(1-\alpha) A(r)+e^{2} A^{2}\right] \\
& \left.-\beta^{2} \pm H(r)\right] g_{n}^{ \pm}\left(r, r^{\prime}\right)=\frac{1}{\alpha r} \delta\left(r-r^{\prime}\right)
\end{aligned}
$$

where $\beta^{2}=k^{2}+\omega^{2}$.

It is of our main interest to investigate the vacuum polarization effect for external points to the magnetic flux. So, we shall consider solutions of (29) with both $r$ and $r^{\prime}$ greater than $R$.

Let us first consider the models $(i)$ and $(i i)$. The inner solution of (29), corresponding to $r<$ $r^{\prime}$ is $g_{n}^{<}\left(r, r^{\prime}\right)$. Integrating out in region $r<R$, we have:

$g_{n}^{ \pm<}\left(r, r^{\prime}\right)=A_{(i)}^{ \pm} H_{i}^{ \pm}(r)$,

for $r<R$ and

$$
\begin{aligned}
g_{n}^{ \pm<}\left(r, r^{\prime}\right)= & B_{(i)}^{ \pm}\left[I_{\left|\nu_{ \pm}\right|}(\beta r)\right. \\
& \left.+E_{(i)}^{ \pm}(\beta R) K_{\left|\nu_{ \pm}\right|}(\beta r)\right],
\end{aligned}
$$

for $R<r<r^{\prime}$, where $\nu_{ \pm}=\frac{\left(n \pm \frac{1-\alpha}{2}-\delta\right)}{\alpha}$, being $\delta=\frac{e \phi}{2 \pi}=N+\gamma \cdot H_{i}^{ \pm}(r)$, for $i=1,2$, represents the solution associated with the two first models:

$H_{1}^{ \pm}(r)=\frac{1}{r} M_{\sigma_{1( \pm)},\left|\lambda_{1( \pm)}\right|}\left(\frac{\delta}{\alpha R^{2}} r^{2}\right)$,

and

$H_{2}^{ \pm}(r)=\frac{1}{\sqrt{r}} M_{\sigma_{2( \pm)},\left|\lambda_{2( \pm)}\right|}(\zeta r)$,

with $\sigma_{1( \pm)}=\left(n-\frac{\beta^{2} R^{2} \alpha^{2}}{2 \delta} \pm \frac{1}{2}(\alpha+1)\right) / 2 \alpha, \lambda_{1( \pm)}=$ $n / 2 \alpha \pm(1-\alpha) / 4 \alpha, \sigma_{2( \pm)}=\frac{\delta(2 n \pm 1)}{2 \alpha\left(\delta^{2}+\beta^{2} \alpha^{2} R^{2}\right)^{1 / 2}}$ and $\lambda_{2( \pm)}=n / \alpha \pm(1-\alpha) / 2 \alpha$. For both functions $H_{2}^{ \pm}, \zeta=\frac{2}{R \alpha}\left(\delta^{2}+\beta^{2} R^{2} \alpha^{2}\right)^{1 / 2}$.

In both cases $M_{\sigma, \lambda}$ represents the Whittaker function. The constant $E_{i}^{ \pm}(\beta R)$ is determined by matching $g_{n}^{ \pm<}$and its first derivative at $r=R$. So we get

$E_{i}^{ \pm}(\beta R)=\frac{S_{i}^{ \pm}(\beta R)}{P_{i}^{ \pm}(\beta R)}$,

where

$S_{i}^{ \pm}(\beta R)=H_{i}^{\prime \pm}(R) I_{\left|\nu_{ \pm}\right|}(\beta R)-H_{i}^{ \pm}(R) I_{\left|\nu_{ \pm}\right|}^{\prime}(\beta R)$

and

$P_{i}^{ \pm}(\beta R)=H_{i}^{ \pm}(R) K_{\left|\nu_{ \pm}\right|}^{\prime}(\beta R)-H_{i}^{\prime \pm}(R) K_{\left|\nu_{ \pm}\right|}(\beta R)$.

In all the expressions, $I_{\nu}$ and $K_{\nu}$ are the modified Bessel functions.

The outer solution of (29) is given by

$g_{n(i)}^{ \pm>}\left(r, r^{\prime}\right)=D_{(i)}^{ \pm} K_{\left|\nu_{ \pm}\right|}(\beta r), \quad$ for $r>r^{\prime}$.

Now imposing the boundary conditions on $g_{n}^{ \pm<}$ and $g_{n}^{ \pm>}$at $r=r^{\prime}$, we get the following result:

$$
\begin{gathered}
g_{n}^{ \pm}\left(r, r^{\prime}\right)=-\frac{1}{\alpha}\left[I_{|\nu \pm|}\left(\beta r^{<}\right)+\right. \\
\left.E^{ \pm}(\beta R) K_{|\nu \pm|}\left(\beta r^{<}\right)\right] K_{|\nu \pm|}\left(\beta r^{>}\right) .
\end{gathered}
$$

Substituting (36) into (28) and after some intermediate steps, the Euclidean Green function acquires the following expression:

$$
\begin{array}{r}
G_{ \pm}\left(x, x^{\prime}\right)=-\frac{e^{i N \Delta \theta}}{8 \pi^{2} \alpha r r^{\prime} \sinh u_{0}} \times \\
\frac{e^{\mp i \Delta \theta} \sinh \left(\gamma^{ \pm} u_{0} / \alpha\right)+\sinh \left[\left(1-\gamma^{ \pm}\right) u_{0} / \alpha\right]}{\cosh \left(u_{0} / \alpha\right)-\cos \Delta \theta}
\end{array}
$$




$$
\begin{array}{r}
-\frac{1}{4 \pi^{2}} \int_{0}^{\infty} d \beta \beta J_{0}\left(\beta \sqrt{(\Delta \tau)^{2}+(\Delta z)^{2}}\right) \times \\
\sum_{n} e^{i n \Delta \theta} E_{n}^{ \pm}(\beta R) K_{\left|\nu_{ \pm}\right|}(\beta r) K_{\left|\nu_{ \pm}\right|}\left(\beta r^{\prime}\right),
\end{array}
$$

with $\gamma^{ \pm}=(1-\alpha) / 2 \pm \gamma$ and

$$
\cosh u_{o}=\frac{r^{2}+r^{\prime 2}+(\Delta \tau)^{2}+(\Delta z)^{2}}{2 r r^{\prime}} .
$$

As to the third model, the solution to $g_{n}^{ \pm}\left(r, r^{\prime}\right)$ is:

$g_{n}^{ \pm<}\left(r, r^{\prime}\right)=A^{ \pm} I_{\left|\nu_{ \pm}\right|}(\beta r)$,

for $r<R$,

$$
\begin{aligned}
g_{n}^{ \pm<}\left(r, r^{\prime}\right)= & B^{ \pm}\left[I_{\left|\epsilon_{ \pm}\right|}(\beta r)\right. \\
& \left.+E^{ \pm}(\beta R) K_{\left|\epsilon_{ \pm}\right|}(\beta r)\right],
\end{aligned}
$$

for $R<r<r^{\prime}$ and

$g_{n}^{ \pm>}\left(r, r^{\prime}\right)=D^{ \pm} K_{\left|\epsilon_{ \pm}\right|}(\beta r)$,

for $r>r^{\prime}$. In the above equation $\epsilon \pm=1 / \alpha(n \pm$ $(1-\alpha) / 2)$. Again the coefficient $A^{ \pm}, B^{ \pm}$and $D^{ \pm}$can be determined by imposing boundary conditions at $r=R$ and $r=r^{\prime}$. However, due the expression to the magnetic field in this model is concentrate as a $\delta$-function at the cylindrical shell, there happens a discontinuity condition in the first derivative of $g^{ \pm<}$at $r=R$. The rest being the same. So using these facts we obtain:

$$
\begin{aligned}
g_{n}^{( \pm)}\left(r, r^{\prime}\right)= & \frac{1}{\alpha}\left[E^{ \pm}(\beta R) K_{\left|\epsilon_{ \pm}\right|}\left(\beta r^{<}\right)\right. \\
& \left.+I_{\left|\epsilon_{ \pm}\right|}\left(\beta r^{<}\right)\right] K_{\left|\epsilon_{ \pm}\right|}\left(\beta r^{>}\right),
\end{aligned}
$$

where now

$E^{ \pm}(\beta R)=\frac{S^{ \pm}(\beta R)}{P^{ \pm}(\beta R)}$

with

$$
\begin{array}{r}
S^{ \pm}(\beta R)=I_{\left|\epsilon_{ \pm}\right|}(\beta R) I_{\left|\nu_{ \pm}\right|}^{\prime}(\beta R)- \\
I_{\left|\nu_{ \pm}\right|}(\beta R) I_{\left|\epsilon_{ \pm}\right| / \alpha}^{\prime}(\beta R) \mp \frac{\delta}{\alpha R} I_{\left|\epsilon_{ \pm}\right|}(\beta R) I_{\left|\nu_{ \pm}\right|}(\beta R)
\end{array}
$$

and

$$
\begin{array}{r}
P^{ \pm}(\beta R)=I_{\left|\nu_{ \pm}\right|}(\beta R) K_{\epsilon_{ \pm}}^{\prime}(\beta R)- \\
I_{\left|\nu_{ \pm}\right|}^{\prime}(\beta R) K_{\left|\epsilon_{ \pm}\right|}(\beta R) \pm \frac{\delta}{\alpha R} K_{\left|\epsilon_{ \pm}\right|}(\beta R) I_{\left|\nu_{ \pm}\right|}(\beta R) .
\end{array}
$$

Finally substituting the expression found to $g_{n}^{ \pm}$ above into (28), and adopting similar procedure as we did in the two previous cases, we obtain:

$$
\begin{gathered}
G_{ \pm}\left(x, x^{\prime}\right)=-\frac{1}{8 \pi^{2} \alpha r r^{\prime} \sinh u_{0}} \times \\
\frac{e^{\mp \Delta \theta} \sinh \left(\bar{\gamma} u_{0} / \alpha\right)+\sinh \left[(1-\bar{\gamma}) u_{0} / \alpha\right]}{\cosh \left(u_{o} / \alpha\right)-\cos \Delta \theta} \\
-\frac{1}{4 \pi^{2}} \int_{0}^{\infty} d \beta \beta J_{0}\left(\beta \sqrt{(\Delta \tau)^{2}+(\Delta z)^{2}}\right) \times \\
\sum_{n=-\infty}^{\infty} e^{i n \Delta \theta} E^{ \pm}(\beta R) K_{\left|\epsilon_{ \pm}\right|}(\beta r) K_{\left|\epsilon_{ \pm}\right|}\left(\beta r^{\prime}\right),
\end{gathered}
$$

with $\bar{\gamma}=(1-\alpha) / 2$.

\section{COMPUTATION OF $\left\langle\hat{T}_{00}\right\rangle_{\text {Ren }}$}

The vacuum expectation value of the energymomentum tensor associated with this system under investigation is given by:

$$
\begin{aligned}
\left\langle\hat{T}_{\mu \nu}\right\rangle= & \frac{1}{4} \lim _{x^{\prime} \rightarrow x} \operatorname{tr}\left[\sigma_{\mu}\left(D_{\nu}-\bar{D}_{\nu^{\prime}}\right)\right. \\
& \left.-\sigma_{\nu}\left(D_{\mu}-\bar{D}_{\mu^{\prime}}\right)\right] S_{F}^{L}\left(x, x^{\prime}\right),
\end{aligned}
$$

where $D_{\sigma}=\nabla_{\sigma}-i e A_{\sigma}$ and the bar denotes complex conjugate and $\sigma^{\mu}=\left(I_{2}, \sigma^{(r)}, \sigma^{(\theta)}, \sigma^{(z)}\right)$.

In order to take into account the presence of the three magnetic field configurations given previously we write the vector potential in the form $A_{\mu}=\left(0,0, \frac{\pi a(r)}{2 \pi \alpha}, 0\right)$, with $a(r)$ being given by (12) and (13), for the first two cases and by (14) for the third case. The spinor Green functions are expressed in terms of the bispinor $G^{L}\left(x, x^{\prime}\right)$ given by (26) with $G_{ \pm}\left(x, x^{\prime}\right)$ given by (37) and (44).

For simplicity let us calculate $\left\langle T_{00}(x)\right\rangle$ only. The other components of the VEV of the energymomentum tensor can be obtained by using the conservation condition and trace anomaly. Fortunately only the second order time derivative provides a nonzero contribution to $\left\langle T_{00}(x)\right\rangle$. In fact all the other terms go to zero in the coincidence limit and/or after taking the trace over the Pauli matrices. Moreover, because the bispinor depends on the time variable with $t-t^{\prime}$, we finally have:

$$
\left\langle T_{00}(x)\right\rangle=-\lim _{x^{\prime} \rightarrow x} \operatorname{tr}\left(\partial_{t}^{2} G\left(x, x^{\prime}\right)\right)
$$




$$
=\lim _{x^{\prime} \rightarrow x} \operatorname{tr}\left(\partial_{\tau}^{2} G_{E}\left(x, x^{\prime}\right)\right),
$$

where we have made a Wick rotation on the above equation.

However the calculation of the above expression provides a divergent result. In order to obtain a finite and well defined result, we must apply in this calculation some renormalization procedure. Here we shall adopt the following prescription: we subtract from the Green function the Hadamard one before applying the coincidence limit as shown:

$$
\begin{aligned}
\left\langle T_{00}(x)\right\rangle_{\text {Ren. } .=} & \lim _{x^{\prime} \rightarrow x} \operatorname{tr}\left[\partial_{\tau}^{2} G_{E}\left(x, x^{\prime}\right)\right. \\
& \left.-\partial_{\tau}^{2} G_{H}\left(x, x^{\prime}\right)\right] .
\end{aligned}
$$

Because this spacetime is locally flat, the Hadamard function coinciding with the Euclidean Green function in a flat spacetime: $G_{H}\left(x, x^{\prime}\right)=$ $\frac{1}{4 \pi} \frac{1}{\left(x-x^{\prime}\right)^{2}} I_{2}$. As it was already mentioned, (37) and (44), present two distinct contributions; the first ones contain informations about the geometrical structure of the spacetime and the fractional part of the magnetic flux, and the second, the corrections, are due to the nonzero thickness of the radius of tube. Moreover, in the calculation of the VEV, only their first contributions are divergent in the coincidence limit, the second are finite. Finally, explicitly exhibiting these remarks, we write down the renormalized VEV of the zerozero component of the energy-moment tensor by:

$\left\langle T_{00}(x)\right\rangle_{\text {Ren. }}=\left\langle T_{00}(x)\right\rangle_{\text {Reg. }}+\left\langle T_{00}(x)\right\rangle_{C}$,

where

$$
\begin{aligned}
\left\langle T_{00}(x)\right\rangle_{\text {Reg. }}= & \lim _{x^{\prime} \rightarrow x}\left[\partial_{\tau}^{2} G_{+}\left(x, x^{\prime}\right)\right. \\
& \left.+\partial_{\tau}^{2} G_{-}\left(x, x^{\prime}\right)-2 \partial_{\tau}^{2} G_{H}\left(x, x^{\prime}\right)\right]
\end{aligned}
$$

and

$$
\left\langle T_{00}(x)\right\rangle_{C}=\lim _{x^{\prime} \rightarrow x} \partial_{\tau}^{2} G_{C}\left(x, x^{\prime}\right),
$$

where, $G_{C}\left(x, x^{\prime}\right)$, represents the corrections due to the second terms in $G_{+}$and $G_{-}$for the three models, as shown next. After some intermediate calculations we arrive to the following results below: i) For the two first models,

$$
\begin{aligned}
& \left\langle T_{00}(x)\right\rangle_{\text {Ren. }}=\frac{1}{5760 \pi \alpha^{4} r^{4}} \times \\
& {\left[\left(\alpha^{2}-1\right)\left(17 \alpha^{2}+7\right)+120 \gamma^{2}\left(\alpha^{2}-2 \gamma^{2}-1\right)\right]} \\
& +\frac{1}{4 \pi^{2} r^{4}} \int_{0}^{\infty} d v v^{3} \sum_{n=-\infty}^{\infty}\left[E_{i}^{+}(v R / r) K_{\left|\nu_{+}\right|}^{2}(v)\right. \\
& \left.+E_{i}^{-}(v R / r) K_{\left|\nu_{-}\right|}^{2}(v)\right],
\end{aligned}
$$

for $i=1$ and 2 .

ii) For the third model,

$$
\begin{aligned}
& \left\langle T_{00}(x)\right\rangle_{\text {Ren. }}=\frac{1}{5760 \pi \alpha^{4} r^{4}}\left(\alpha^{2}-1\right)\left(17 \alpha^{2}+7\right) \\
& +\frac{1}{4 \pi^{2} r^{4}} \int_{0}^{\infty} d v v^{3} \sum_{n=-\infty}^{\infty}\left[E^{+}(v R / r) K_{|\epsilon+|}^{2}(v)\right. \\
& \left.+E^{-}(v R / r) K_{|\epsilon-|}^{2}(v)\right] .
\end{aligned}
$$

For all the above expressions the coefficient $E^{ \pm}$ were given in (34) and (43).

Before to provide some qualitative information about the second contributions of (51) and (52), we would like to make a few comments about our results: (i) The first contributions of them depend only on the conicity parameter $\alpha$, and the fractional part of $\phi / \phi_{0}$, denoted by $\gamma$, for the two first models. (ii) The second contributions, the corrections, vanish in the limit $R \rightarrow 0$. They also depend on the integer part of $\phi / \phi_{0}, N$.

Some qualitative information about the behavior of the second contributions of (51) and (52) can be provided. Although for the three models these contributions present an overall $1 / r^{4}$ dependence on the radial coordinate, there exist an additional dependece in their integrand by the coefficient $E^{ \pm}$. By our numerical analysis each term present opposite signal; however they do not cancel each other. Our analysis were developed for $N=0$ and $N=1$, for $\alpha=0.99, \gamma=0.02$ and $R / r=10^{-3}$.

\section{CONCLUDING REMARKS}

In this paper we have explicitly exhibited the spinor Green functions associated with a charged left-handed field on a cosmic string spacetime in the presence of external magnetic fluxes in three 
different configurations, all of them confined in a long tube of radius $R$. Having these Green functions we calculated the formal expression to the renormalized VEV of the zero-zero component of energy-momentum tensor, $\left\langle T_{00}(x)\right\rangle_{\text {Ren }}$. In these calculations we observe that two independent contributions were obtained. The first contributions are the standard ones due to the conical geometry of the spacetime and the magnetic flux. They coincide with the expression found by Linet in [3]. The second contributions are corrections due to the finite thickness of the radius of the tube. Unfortunately, it was not possible to obtain an analytical expression to provide the dependence of these second contributions with the radial coordinate, and only by numerical analysis we can do this. However some qualitative information can be given: we found for specific values of the parameter that the integrands of these corrections have opposite signal, however they do not cancel each other. Our next task will be to develop a more systematic numerical analysis to provide quantitative information about the second parts of (51) and (52).

Acknowledgments

We would like to thank Conselho Nacional de Desenvolvimento Científico e Tecnológico (CNPq.) and CAPES for partial financial support.

\section{REFERENCES}

1. E. M. Serebryanyi, Theor. Math. Phys. 64, 846 (1985).

2. M. E. X. Guimarães and B. Linet, Commu. Math. Phys. 165, 297 (1994).

3. B. Linet, J. Math. Phys. 36, 3694 (1995).

4. L. Sriramkumar, Class. Quantum Grav. 18, 1015 (2001).

5. J. Spinelly and E. R. Bezerra de Mello, Class. Quantum Grav. 20, 873 (2002); J. Spinelly and E. R. Bezerra de Mello, Int. J. Mod. Phys. A 17, 4375 (2002).

6. N. D. Birrel and P. C. W. Davies, Quantum Filed in Curved Space (Cambridge University Press. Cambridge, England, 1982). 